\title{
Even-odd effects in the prompt fission emission of even $Z$ actinides
}

\author{
Anabella Tudora ${ }^{1, a}$, Franz-Josef Hambsch ${ }^{2}$, Georgiana Giubega ${ }^{1}$ and Iuliana Visan ${ }^{1}$ \\ ${ }^{1}$ University of Bucharest, Faculty of Physics, 405 Atomistilor Str., POB MG-11, Magurele 77125, Romania \\ ${ }^{2}$ EC-JRC-Institute for Reference Materials and Measurements (IRMM), Retieseweg 111, Geel, 2440 Belgium
}

\begin{abstract}
The investigation of even-odd effects in the prompt emission of even $Z$ actinides showed a sawtooth shape of $v(Z)$ with staggering in the asymmetric fission region. Average prompt emission quantities as a function of A, e.g. v(A), of even $\mathrm{Z}$ fragmentations are higher than those of odd $\mathrm{Z}$ fragmentations and they exhibit oscillations with a periodicity of about 5 mass units in the asymmetric fission region. This periodicity is not due to the $Z$ even-odd effect in fragment distributions. The even-odd effect in $\langle v>$ (TKE) is increasing with increasing TKE and it decreases with increasing mass of the fissioning nucleus. The global even-odd effect in total average prompt emission quantities is decreasing with increasing mass of the fissioning nucleus. In the case of an even-odd fissioning nucleus, ${ }^{234} \mathrm{U}(\mathrm{n}, \mathrm{f})$, the global even-odd effect in prompt emission quantities exhibits a very slow variation with the incident neutron energy.
\end{abstract}

\section{Introduction}

Among the characteristics of fission fragment distributions the $\mathrm{Z}$ even-odd effect has repeatedly come into attention as an interesting feature because it contains information about the saddle to scission process, playing an important role in the fission modelling. The neutron even-odd effect received less attention since only the charge number $\mathrm{Z}$ and the post neutron mass number are known experimentally with sufficient accuracy. Also it should be kept in mind that due to the even-odd staggering in neutron separation energy, the prompt neutron evaporation brings an even-odd effect of its own. Contrary to the even-odd effects in fission fragment distributions that were extensively studied, the even-odd effects in the prompt neutron and $\gamma$-ray emission received less attention. Our recent works focused on this subject. The first studies referred to the even-odd effects in the prompt emission of even-even actinides fissioning spontaneously, ${ }^{252} \mathrm{Cf}(\mathrm{SF}),{ }^{236-244} \mathrm{Pu}(\mathrm{SF})$ and induced by thermal neutrons ${ }^{233,235} \mathrm{U}\left(\mathrm{n}_{\mathrm{th}}, \mathrm{f}\right),{ }^{239} \mathrm{Pu}\left(\mathrm{n}_{\mathrm{th}}, \mathrm{f}\right)$. The results are reported in Refs. [1, 2]. The study is continued with an evenodd fissioning nucleus, ${ }^{234} \mathrm{U}(\mathrm{n}, \mathrm{f})$ at incident neutron energies (En) ranging from $0.2 \mathrm{MeV}$ to $5 \mathrm{MeV}$. Because the fragment distributions play an important role in the calculation of different average prompt emission quantities as a function of $\mathrm{A}$, of $\mathrm{Z}$, of TKE and total average ones, it is also interesting to see the impact on the prompt emission of the following basic features of the $\mathrm{Z}$ even-odd effect in experimental fragment distributions (mentioned in the majority of publications, e.g. [3, 4] and references therein): i) the effect globally decreases for heavier fissioning systems (some authors mentioning that decreases with the fissility parameter), ii) the effect grows with the fragmentation

\footnotetext{
${ }^{\text {a }}$ Corresponding author: anabellatudora@hotmail.com
} 
asymmetry, iii) the amplitude of the even-odd structure decreases with increasing excitation energy of the fissioning nucleus, iv) the $\mathrm{Z}$ even-odd effect grows with the fragment kinetic energy.

The Point-by-Point $(\mathrm{PbP})$ model of prompt emission provides as primary results the multiparametric matrices of different quantities characterizing both the fragments and the prompt emission, generically labelled q(A,Z,TKE) (e.g. v(A,Z,TKE), E $\gamma(\mathrm{A}, \mathrm{Z}, \mathrm{TKE}), \mathrm{a}(\mathrm{A}, \mathrm{Z}, \mathrm{TKE})$ ). Different average quantities $q(Z), q(A), q(T K E)$ and $<q>$ are obtained by averaging the $\mathrm{PbP}$ matrices $\mathrm{q}(\mathrm{A}, \mathrm{Z}, \mathrm{TKE})$ over the multiple distributions $\mathrm{Y}(\mathrm{A}, \mathrm{Z}, \mathrm{TKE})$ in different ways (for details see Refs. $[1,2,5,6]$ and references therein). These distributions are taken as $Y(A, Z, T K E)=Y(A, T K E) p(Z, A)$ in which $\mathrm{Y}(\mathrm{A}, \mathrm{TKE})$ are experimental distributions (usually reconstructed from the single ones $\mathrm{Y}(\mathrm{A}), \mathrm{TKE}(\mathrm{A})$ and $\left.\sigma_{\mathrm{TKE}}(\mathrm{A})\right)$ and the isobaric charge distributions $\mathrm{p}(\mathrm{Z}, \mathrm{A})$ are provided by the Zp model of Wahl [7-9]. The global even-odd effect in $\mathrm{Y}(\mathrm{Z})$ projections, defined according to Gönnenwein [3]:

$$
\delta_{Y(Z)}=\left(\sum_{Z e} Y(Z e)-\sum_{Z o} Y(Z o)\right) / \sum_{Z} Y(Z)
$$

as well as the even-odd effects in the charge polarization $\Delta \mathrm{Z}(\mathrm{A})=\mathrm{Zp}(\mathrm{A})-\mathrm{Z}_{\mathrm{UCD}}(\mathrm{A})$ and the root-meansquare $\operatorname{rms}(A)$ of the Gaussian functions fitting the isobaric charge distribution $p(Z, A)$ are investigated. The charge polarisation plays an important role in the construction of the fragmentation range of the $\mathrm{PbP}$ treatment. I.e. at each $\mathrm{A}$ (covering a large range from symmetric fission up to a very asymmetric split) 3 up to 5 charge numbers $Z$ being taken as the nearest integers above and below the most probable charges $\mathrm{Zp}(\mathrm{A})$ (that are the values on which the fitting Gaussians are centred). TKE covers a large range (usually from $100 \mathrm{MeV}$ to $200 \mathrm{MeV}$ with a step of $5 \mathrm{MeV}$ ).

We have investigated the global $\mathrm{Z}$ and $\mathrm{N}$ even-odd effects in different total average quantities characterizing the prompt emission, defined as $[1,2]$ :

$$
\delta_{<q>}=\left(\langle q\rangle_{\text {even } Z / N}-\langle q\rangle_{\text {odd } Z / N}\right) /\langle q\rangle
$$

The behaviour of average $\mathrm{q}(\mathrm{Z})$, of average $\mathrm{q}(\mathrm{A})$ of even $\mathrm{Z}$ and odd $\mathrm{Z}$ fragmentations and of average neutron separation energy $<\mathrm{Sn}>$ as a function of $\mathrm{N}$ were investigated, too. The variation of the $\mathrm{Z}$ evenodd effect in different average quantities as a function of TKE is emphasized by the function $[1,2]$ :

$$
\delta_{<q>}(T K E)=\left(<q>_{\text {even }}(T K E)-<q>_{\text {odd } Z}(T K E)\right) /<q>(T K E)
$$

Note, cold fission is coming close to neutron-less fission. Because this work is referring to prompt neutron emission, cold fission fragmentations are not taken into account. To avoid any limit situation the fragmentations with TXE less than $9 \mathrm{MeV}$ were excluded (see Refs. [1,2] and references therein).

\section{Even-odd effects in the prompt emission of even $Z$ actinides}

\subsection{Even-even nuclei fissioning spontaneously or induced by thermal neutrons}

As it was reported in $[1,2]$ the global even-odd effect in the $Y(Z)$ projections is decreasing with increasing mass of the fissioning system: from about $22 \%$ for ${ }^{235,233} \mathrm{U}\left(\mathrm{n}_{\mathrm{th}}, \mathrm{f}\right)$ to about $10-14 \%$ for eveneven $\mathrm{Pu}$ isotopes fissioning spontaneously and by thermal neutrons and up to about $4 \%$ for ${ }^{252} \mathrm{Cf}(\mathrm{SF})$. $\Delta \mathrm{Z}(\mathrm{A})$ and $\mathrm{rms}(\mathrm{A})$ exhibit oscillations with a periodicity of about 5 mass units in the asymmetric fission region. The oscillation amplitudes are also decreasing with increasing mass of the fissioning system, they being the most pronounced for ${ }^{233,235} \mathrm{U}\left(\mathrm{n}_{\mathrm{th}}, \mathrm{f}\right)$ and almost vanished in the case of ${ }^{252} \mathrm{Cf}(\mathrm{SF})$.

These features of even-odd effects in fragment distributions and charge polarizations are reflected in the prompt emission. In all cases $v(Z)$ exhibit sawtooth shapes and a visible staggering in the asymmetric fission region. In the case of ${ }^{252} \mathrm{Cf}(\mathrm{SF})$ the $v(\mathrm{Z})$ staggering is visibly less pronounced compared to $\mathrm{U}$ and $\mathrm{Pu}$ isotopes. The average quantities as a function of A (e.g. TXE(A), v(A), E $\gamma(\mathrm{A})$ ) of even $\mathrm{Z}$ fragmentations are higher than those of odd $\mathrm{Z}$ fragmentations and both exhibit oscillations with a periodicity of about 5 mass units. This periodicity is not due to the $Z$ even-odd effects in fragment distributions. Only the magnitude of oscillation amplitudes reflects the size of the effect. Average quantities as a function of TKE of even $\mathrm{Z}$ fragmentations are also higher than those of odd $\mathrm{Z}$ ones. The function of Eq.(3) is plotted in Fig.1. In the case of ${ }^{235,233} \mathrm{U}\left(\mathrm{n}_{\mathrm{th}}, \mathrm{f}\right),{ }^{239} \mathrm{Pu}\left(\mathrm{n}_{\mathrm{th}}, \mathrm{f}\right)$ and ${ }^{252} \mathrm{Cf}(\mathrm{SF})$ 
(upper part) the increase of the even-odd effect in prompt neutron multiplicity with TKE and the decrease of this effect for heavier fissioning systems is visible. In the case of ${ }^{236-244} \mathrm{Pu}$ (SF) (lower part) the even-odd effect is also increasing with TKE but the decrease of the effect for heavier fissioning nuclei is not visible.

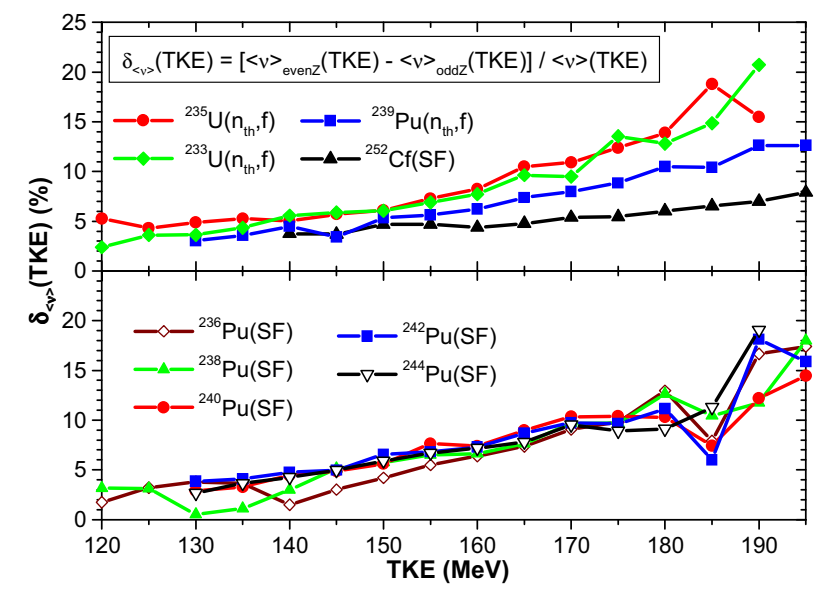

Figure 1. The even-odd effect in prompt neutron multiplicity as a function of TKE of ${ }^{233,235} \mathrm{U}\left(\mathrm{n}_{\mathrm{th}}, \mathrm{f}\right),{ }^{239} \mathrm{Pu}\left(\mathrm{n}_{\mathrm{th}}, \mathrm{f}\right)$ and ${ }^{252} \mathrm{Cf}(\mathrm{SF})$ in the upper part and of ${ }^{236,238,240,242,244} \mathrm{Pu}(\mathrm{SF})$ in the lower part.

The global even-odd effect (given by Eq.(2)) in $\langle$ TXE $>,<v>$ and other quantities is decreasing with increasing mass of the fissioning system, e.g. $\delta<v>$ is of about $9 \%$ for ${ }^{233,235} \mathrm{U}\left(\mathrm{n}_{\mathrm{th}}, \mathrm{f}\right)$, of about $8 \%$ for ${ }^{239} \mathrm{Pu}\left(\mathrm{n}_{\mathrm{th}}, \mathrm{f}\right)$ and $6 \%$ for ${ }^{252} \mathrm{Cf}(\mathrm{SF})$. In the case of $\mathrm{Pu}(\mathrm{SF}) \delta<\nu>$ is almost constant (of about $7-8 \%$ ). In all cases the global even-odd effect in the average prompt neutron energy $<\mathrm{E}>$ and average prompt $\gamma-$ ray energy $\langle\mathrm{E} \gamma\rangle$ is $2.5-3$ times lower than the effect in prompt neutron multiplicity. Details about the even-odd effect in the prompt emission of the thermal neutron induced fission of ${ }^{233,235} \mathrm{Uand}^{239} \mathrm{Pu}$ and ${ }^{252} \mathrm{Cf}(\mathrm{SF})$ are given in Ref. [1] and of ${ }^{236-244} \mathrm{Pu}(\mathrm{SF})$ in Ref.[2].

\subsection{Even-odd fissioning nuclei: ${ }^{234} \mathrm{U}(\mathrm{n}, \mathrm{f})$ at En ranging from $0.2 \mathrm{MeV}$ to $5 \mathrm{MeV}$}

The experimental $Y(A, T K E)$ distributions of ${ }^{234} \mathrm{U}(\mathrm{n}, \mathrm{f})$ measured at IRMM at 14 incident energies ranging from $0.2 \mathrm{MeV}$ to $5 \mathrm{MeV}$ and the $\mathrm{Zp}$ model with parameters from systematics, mainly developed by Wahl $[8,9]$, allow the investigation of even-odd effects. The even-odd factors $F_{Z}$ and $F_{N}$ entering the $\mathrm{Zp}$ model play the most important role. Based on existing systematics of these factors [10] several parameterisations (showing different decreases of $\mathrm{F}_{Z}$ and $\mathrm{F}_{\mathrm{N}}$ with increasing En) can be deduced. They led to different $\mathrm{Y}(\mathrm{A}, \mathrm{Z}, \mathrm{TKE})$ distributions and charge polarizations. The obtained global even-odd effects in $Y(Z)$ at the 14 incident energies (according to Eq.(1)) are plotted with different symbols in the left part of Fig.2. The uncertainties in $\delta_{\mathrm{Y}(\mathrm{Z})}$ resulting from the propagation of uncertainties in the experimental distributions are included in the size of symbols. As it can be seen $\delta_{\mathrm{Y}(\mathrm{Z})}$ exhibits a clear decrease with En in all cases. The linear fits to all $\delta_{\mathrm{Y}(\mathrm{Z})}$ are plotted with dashed lines in the same colour as the respective symbol. The obtained $\delta_{\mathrm{Y}(\mathrm{Z})}$ values, varying from about $26 \%$ (the highest result) to about $4-5 \%$ at $5 \mathrm{MeV}$ are in overall agreement with the compilation of Ref. [11] giving an even-odd effect of $(23 \pm 8.9) \%$ for the fission of ${ }^{234} \mathrm{U}$ induced by neutrons with fission spectrum energies. Our $\mathrm{Y}(\mathrm{Z})$ results (plotted with red circles and blue squares in the right part of Fig.2) describe well the experimental data of the electromagnetic induced fission of ${ }^{235} \mathrm{U}$ obtained in the SOFIA experiment at GSI [12] (black stars connected with a thick line) in the asymmetric fission region. The differences appearing at symmetric fission are due to the different ways to induce the fission of ${ }^{235} \mathrm{U}$.

The two facts illustrated in Fig.2 (the $\delta_{\mathrm{Y}(\mathrm{Z})}$ results in overall agreement with the compilation of Ref. [11] and the good description of the $\mathrm{Y}(\mathrm{Z})$ data of ${ }^{235} \mathrm{U}(\mathrm{em} . \mathrm{f})$ ) can be considered as a validation of 
the $\mathrm{Y}(\mathrm{A}, \mathrm{Z}, \mathrm{TKE})$ distributions based on experimental $\mathrm{Y}(\mathrm{A}, \mathrm{TKE})$ data and the Zp model with parameters from systematics.
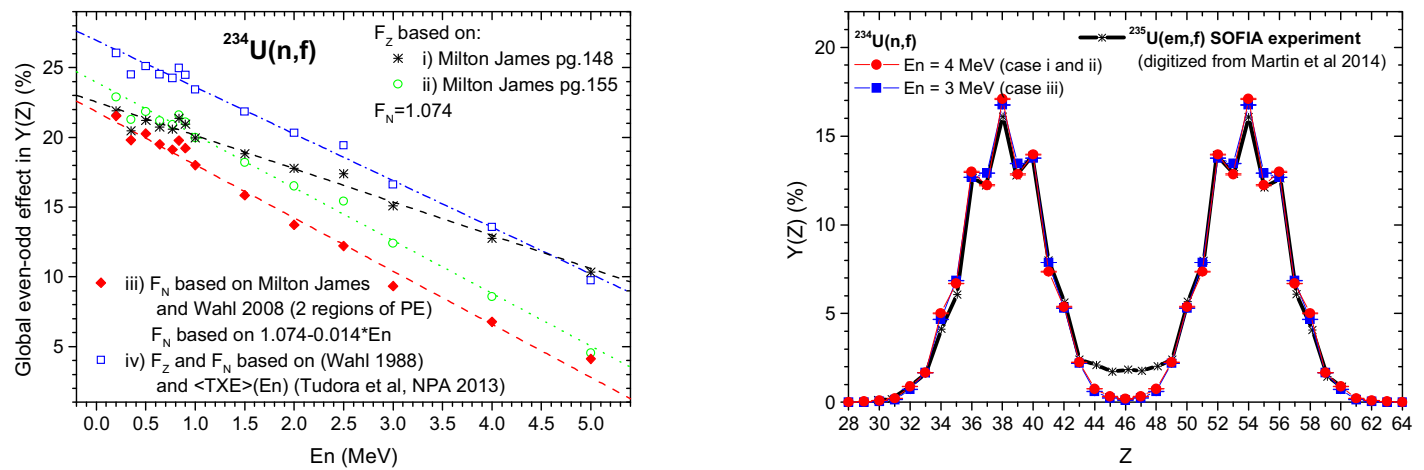

Figure 2: Left part: the global even-odd effect in $Y(Z)$ as a function of En obtained with different $F_{Z}$ and $F_{N}$ parameterisations based on existing systematics. Right part: comparison of present $\mathrm{Y}(\mathrm{Z})$ results of ${ }^{234} \mathrm{U}(\mathrm{n}, \mathrm{f})$ (red circles and blue squares) with the experimental data of ${ }^{235} \mathrm{U}(\mathrm{em} . \mathrm{f})$ (SOFIA experiment at GSI) given with black stars connected with a thick line.

The impact of different prescriptions of $\mathrm{Zp}$ model parameters on the even-odd effects in prompt emission was also investigated. The differences between the probabilities of even $\mathrm{Z}$ and odd $\mathrm{Z}$ fragmentations able to emit prompt neutrons given by different $\mathrm{Zp}$ model parameter prescriptions are low, of about $2-3 \%$. This fact leads to insignificant differences between the global even-odd effects in different prompt emission quantities (e.g. $\langle v\rangle,\langle\mathrm{TXE}\rangle,\langle\mathrm{E} \gamma\rangle$ ) obtained with different prescriptions of $\mathrm{Zp}$ model parameters (the differences being less than the uncertainties in the global even-odd effects).

The global even-odd effects in different prompt emission quantities show a very slowly decreasing trend with increasing En. Practically they can be considered as constant with En. E.g. $\delta<v>$ is varying between $8 \%-9 \%$, being at the same level of magnitude as in the case of neighbouring even-even nuclei ${ }^{235,233} \mathrm{U}(\mathrm{nth}, \mathrm{f})$. This slow variation with En is due to a) the $\mathrm{N}$ even-odd staggering in $<\mathrm{Sn}>$, the prompt neutron emission being favoured for odd $\mathrm{N}$ fragments and $\mathrm{b}$ ) the even-odd character of the fissioning nucleus leading to pairs with even $\mathrm{N}$ and odd $\mathrm{N}$ complementary fragments. The favoured prompt emission for odd $\mathrm{N}$ fragments is often acting contrary to the $\mathrm{Z}$ even-odd effect in fragment distributions (higher probabilities of even $\mathrm{Z}$ pairs than of odd $\mathrm{Z}$ pairs).

The even-odd effects in different average quantities as a function of $Z$, of $A$, of TKE have the same characteristics as those of even-even fissioning nuclei. E.g. $v(Z)$ exhibit a sawtooth shape and staggering in the asymmetric fission region. The interesting behaviour observed experimentally in the case of $v(\mathrm{~A})$, consisting in the multiplicity increase with En mainly for heavy fragments, is exhibited by $v(Z)$, too. Different average quantities as a function of A (e.g. $v(A)$, TXE(A)) of even $Z$ fragmentations are higher than those of odd $Z$ fragmentations. Their oscillation periodicity of about 5 mass units is not due to the $Z$ even-odd effects in fragment distributions.

The increase of the $\mathrm{Z}$ even-odd effect in different quantities with increasing TKE, expressed by the function of Eq.(3), is illustrated in Fig.3 for the prompt neutron multiplicity. The very low even-odd effect at low TKE values can be explained by the behaviour of the experimental Y(A,TKE) distributions as a function of A. At low TKE values the yields Y(A.TKE) are high in the mass region near symmetric fission where the even-odd effect in $Z$ is very low. In terms of multi-modal fission here the symmetric super-long mode SL is dominant. At medium TKE values the yields are high in the mass region of asymmetric fission (with maximums placed at $A_{H}$ of about 140-145) where the $Z$ even-odd effect is pronounced. Here the fission mode S2 is the dominant one. At high TKE values the yields are high in the mass region of asymmetric fission, too (with maximums placed at $\mathrm{A}_{\mathrm{H}}$ of about 132-135) where the even-odd effect is pronounced. At high TKE the fission mode S1 is dominant. 


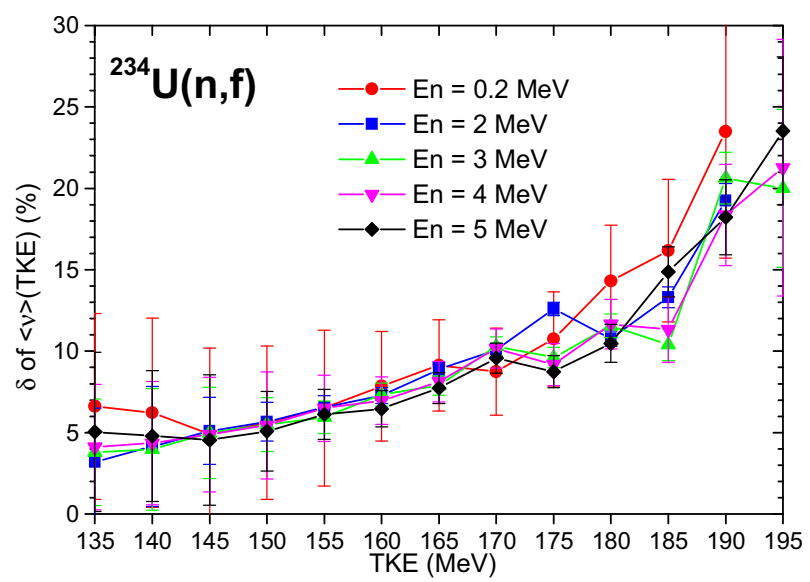

Figure 3. Even-odd effect in prompt neutron multiplicity as a function of TKE (expressed by Eq.(3)) at a few En ranging from $0.2 \mathrm{MeV}$ to $5 \mathrm{MeV}$.

\section{Conclusions}

In the case of ${ }^{234} \mathrm{U}(\mathrm{n}, \mathrm{f})$ even if different prescriptions of the $\mathrm{Zp}$ model parameters led to differences in the even-odd effects in $\mathrm{Y}(\mathrm{Z})$ and $\Delta \mathrm{Z}(\mathrm{A})$ insignificant differences in the global even-odd effects in prompt emission were obtained, due to the low differences in the probabilities of even $\mathrm{Z}$ and odd $\mathrm{Z}$ fragmentations able to emit prompt neutrons provided by different $\mathrm{Zp}$ model parameter prescriptions.

Even if the global even-odd effect in $\mathrm{Y}(\mathrm{Z})$ is decreasing with increasing En, the global even-odd effects in different total average prompt emission quantities shows a very slow variation with En.

$v(Z)$ exhibits a sawtooth shape with staggering in the asymmetric fission region. It increases with En mainly for heavy fragments as in the case of $v(\mathrm{~A})$. The $Z$ even-odd effect in $\langle v\rangle$ (TKE) is increasing with TKE. The very low even-odd effect at low TKE values is explained by the behaviour of the Y(A,TKE) yields as a function of A for low, medium and high TKE values in correlation with the presence of the even-odd effects in the asymmetric fission region.

\section{References}

1. A.Tudora, F.-J.Hambsch, G.Giubega, I.Visan, Nucl.Phys.A 929, 260-292 (2014)

2. A.Tudora, F.-J.Hambsch, G.Giubega, I.Visan, Nucl.Phys.A 933, 165-188 (2015)

3. F.Gonnenwein in The Nuclear Fission Process (CRC Press Boca Raton NY USA) ch. 8 (1991)

4. N.Caamano, F.Rejmund, K.-H.Schmidt, J.Phys.G Nucl.Part.Phys. 38035101 (2011)

5. A.Tudora, F.-J.Hambsch, I.Visan, G.Giubega, Nucl.Phys.A 940, 242-263 (2015)

6. A.Tudora, F.-J.Hambsch, S.Oberstedt, Nucl.Phys.A 917, 43-70 (2013)

7. A.C.Wahl, At.Data Nucl.Data Tables 39, 1-156 (1988).

8. A.C.Wahl in Fission Product Yield Data for the Transmutation of Minor Actinide Nuclear Waste (IAEA STI/PUB/1286) chapter 4.2 (2008)

9. A.C.Wahl in Compilation and evaluation of fission yield nuclear data (IAEA TECDOC-1168) Sections 4.1, 4.2, 7.4 (2000)

10. R.W.Mills, M.F.James in Compilation and evaluation of fission yield nuclear data (IAEA TECDOC-1168) Section 7.2 (2000)

11. T.R.England, B.F.Rider Evaluation and compilation of fission product yields LA-U-94-3106 ENDF-349 (1993)

12. J.F.Martin for the SOFIA collaboration, $P(N D)-2$ International workshop on perspectives of nuclear data for the next decade (organised by CEA and NEA) Bruyères-le-Châtel, France (2014) 
